\title{
On the Influence of Weather Forecast Errors in Short-Term Load Forecasting Models.
}

\author{
D. Fay* J.V. Ringwood ${ }^{\dagger}$ M. Condon \\ *NUI Galway, Galway, Ireland. fax: +353 (091) 750542 email: damien.fay@nuigalway.ie †NUI Maynooth, Co. Kildare, \\ Ireland. ‡ DCU, Dublin 9, Ireland.
}

Keywords: Load forecasting, weather forecast errors, model combination, data fusion.

\begin{abstract}
Weather information is an important factor in load forecasting models. This weather information usually takes the form of actual weather readings. However, online operation of load forecasting models requires the use of weather forecasts, with associated weather forecast errors. A technique is proposed to model weather forecast errors to reflect current accuracy. A load forecasting model is then proposed which combines the forecasts of several load forecasting models. This approach allows the relationship between weather and load to be determined without weather forecast error. The effect of the weather forecast error is then minimised during the combination stage.
\end{abstract}

\section{Introduction}

Short Term Load Forecasting (STLF) refers to forecasts of electricity demand (or load), on an hourly basis, from one to several days ahead. The amount of excess electricity production (or spinning reserve) required to guarantee supply, in the event of an underestimation, is determined by the accuracy of these forecasts. Conversely, overestimation of the load leads to sub-optimal scheduling (in terms of production costs) of power plants (unit commitment). In accordance with the Electricity Regulation Act of 1999, a deregulated market structure was set up in Ireland, which should lead to increased impetus to reducing forecast error and the associated costs.

As illustrated above, STLF is an important area and this is reflected in the literature by the many techniques that have been applied, including neural networks [7], fuzzy logic [9] and statistical techniques [12], to mention but a few. In many electricity grid systems, the prevailing weather has a significant effect on the load and it has been found that including weather information can improve a load forecast $[19,15]$. However, in order to use weather information for future load forecasts, weather forecasts must be utilised and these have associated weather forecast errors. Although system dependent, weather forecast errors can be significant and have been attributed as the cause of $17 \%$ [5] to $60 \%$ [8] of load forecast errors.

Load forecasting models are usually trained using actual past weather readings [14] as opposed to past weather forecasts.
This is based on the assumption that to use the latter essentially adds forecast noise to the training data. Often weather forecasts are unavailable for the entire training period and/or can be subject to increasing accuracy of meteorological models, as mathematical weather models are constantly improved. Therefore, training load models with actual weather can be justified [14]. However, when weather forecast errors not present in the training set are presented, they can have a disproportionate influence on load models $[21,4]$. Changing the load model parameters to account for this can be impossible in many conventional models once training is completed. Douglas et al. [5] approached this problem by use of a Bayesian framework, but restricted analysis to the use of dynamic linear models. In spite of the importance of weather forecast errors with respect to load forecasting, the literature is sparse [11].

This paper proposes combining several models (called submodels), or model fusion, as a technique for minimising the effect of weather forecast errors in load forecasting models. The concept of model fusion is well known in the general field of forecasting and was pioneered mainly in $[1,17]$. Fused forecasts are often more accurate than any of the individual model forecasts $[13,20]$ as different models are often better at modelling different aspects of an underlying process and thus combining the models appropriately gives a better forecast. In addition, a single model incorporating all aspects of an underlying process may be more complex and difficult to train than combining individual models [13]. Model fusion has been applied to many fields [20, 18]. Model fusion is particularly suited to STLF as the sub-models may be trained with actual weather information and the effect of weather forecast errors taken into account when combining the models.

\section{Data set details}

The range and time-scale of the available electrical demand data is given in Table 1.

\begin{tabular}{|l|l|}
\hline Range & $29 / 12 / 1986-31 / 03 / 2000$ \\
\hline Time scale & Hourly \\
\hline No. of data points & 4842 Days (116208 hours) \\
\hline
\end{tabular}

Table 1: ESB data time-scale and range. 
Two categories of historical weather data are available from the Meteorological Office of Ireland (MOI): readings (or actual weather) and forecasts. Both sets of data are for Dublin airport, the closest and most relevant weather station to Dublin (Table 2). The readings and forecasts are for dry bulb temperature, cloud cover, wind speed and wind direction.

\begin{tabular}{|l|l|l|}
\hline Type & Range & $\begin{array}{l}\text { Time } \\
\text { scale }\end{array}$ \\
\hline Weather readings & $29 / 12 / 1986-$ & Hourly \\
& $31 / 03 / 2000$ & \\
Weather forecasts & $01 / 02 / 2000-$ & Hourly \\
& $01 / 03 / 2000$ & \\
\hline
\end{tabular}

Table 2: Weather data time-scale and range.

The data is subdivided into three sets in order to train and test the load forecasting models (Table 3 ). The training set is used to calculate model parameters, the validation set is used to aid in model structure determination and the novelty set is used to evaluate model performance.

\begin{tabular}{|l|l|l|l|}
\hline Set & Training & Validation & Novelty \\
\hline Range & $1987-1997$ & 1998 & $1999-2000$ \\
\hline
\end{tabular}

Table 3: Division of data set.

Data between Monday and Friday in the months January to March is selected so as to avoid the exceptions associated with weekend, Christmas and changes due to the daylight saving hour.

\section{Modelling weather forecast errors.}

Due to the sparseness of weather forecast data (Table 2) it is necessary to model the weather forecast error to produce pseudo-weather forecasts for the entire data set. Previous approaches in STLF have modelled the weather forecast error as a Gaussian random variable [16,3]. However, as seen in Figure 1 this is not an accurate representation of the statistics of the weather forecast errors in Ireland. Rather, the forecast error is either above or below the actual for prolonged periods. Typically some form of aggregate weather variables are normally used in STLF models (e.g. average daily temperature). The error in an aggregate weather variable will have a non-zero mean (Figure 1) and a Gaussian approximation in would underestimate this.

The weather in Ireland is dominated by Atlantic weather systems. When a weather system or front reaches Ireland there is a shift in the level of the temperature and other weather variables (Figure 1). This shift is also a factor that the Irish Meteorological Office must forecast. The weather forecast error is thus presumed to have the following structure:

- $\quad$ Turning points (Figure 1) which represent the arrival of a weather front,
- A level error, $\tilde{\mu}$, which is the average of the weather forecast error between turning points,

- A shape error, $\tilde{\sigma}$, which is the standard deviation of the weather forecast error between turning points, and

- A random error, which accounts for the remaining error if $\widetilde{\mu}$ and $\widetilde{\sigma}$ are removed.

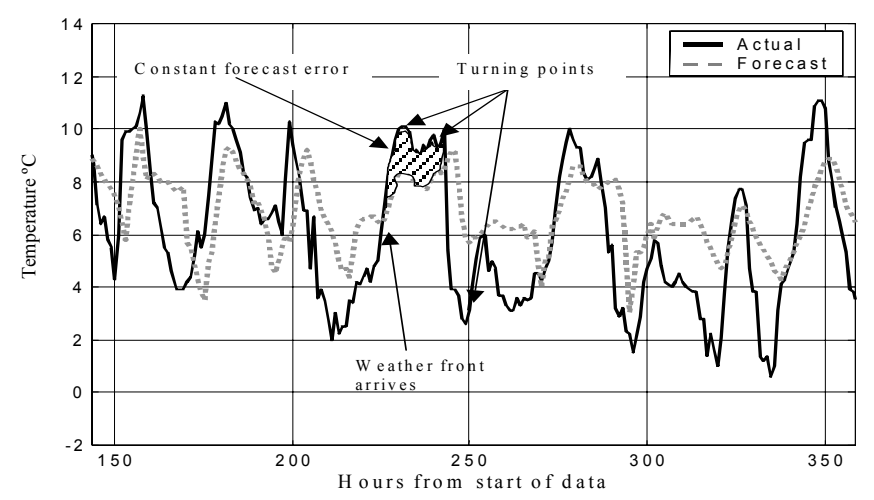

Figure 1. Actual and forecast temperature $\left(6^{\text {th }}\right.$ to $15^{\text {th }}$ February 2000).

The turning points were found by using a peak detection algorithm. Also, the distributions of $\widetilde{\mu}, \widetilde{\sigma}$ and the random error are found to be approximately Gaussian for the four weather variables used. In addition, all the shape and level errors of the four weather variables are cross correlated, suggesting that they may be jointly distributed.

In order to generate pseudo-weather forecast errors, the turning points in the actual weather variables are first identified. Then, a multivariate Gaussian pseudo-random number generator is used to generate $\tilde{\mu}, \tilde{\sigma}$ and the random errors for each the weather variables jointly. Figure 2, below, shows the Sample Auto-Correlation Function (SACF) [2] of the temperature forecast errors and the pseudo-temperature forecast errors. As can be seen, the SACF for both are similar, showing that the pseudo-forecast errors has captured the autocorrelation evident in the temperature forecast errors. A similar situation was found with the other weather variables.

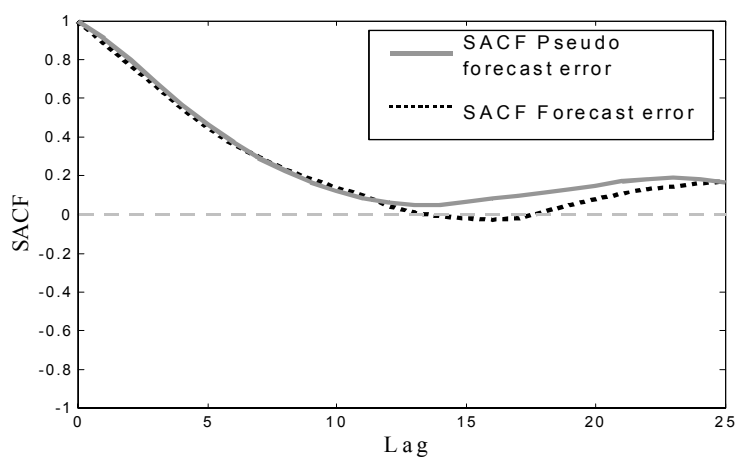

Figure 2. SACF of forecast and pseudo-forecast temperature errors. 


\section{The fusion model}

\subsection{Preliminary Auto-Regressive (AR) linear model}

It was previously found by these authors [6] that decomposing load data into 24 parallel series, one for each hour of the day, is advantageous as the parallel series have a degree of independence. The parallel series for hour $j$ on day $k, y(j, k)$, has a low frequency trend, $d(j, k)$, which is first removed using a Basic Structural Model (BSM) leaving a residual, $x(j, k)$, (Figure 3) which is composed of weather, non-linear auto-regressive and white noise components [6].

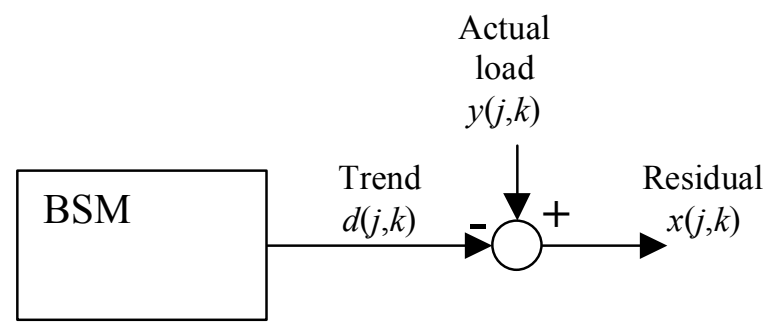

Figure 3. Preliminary AR linear model overview

\subsection{Sub-Models}

Three models were chosen which have different types of inputs. These are chosen so that forecast errors can be attributed to particular inputs. A fourth model is included using all the available inputs to capture any non-linear relationships between the inputs and the residual. The fusion technique combines the forecasts of the sub-models $x_{1}(j, k), \ldots, x_{4}(j, k)$ to give a fused forecast, $x_{f}(j, k)$, of the residual for series $j$ on day $k$ (Figure 4).

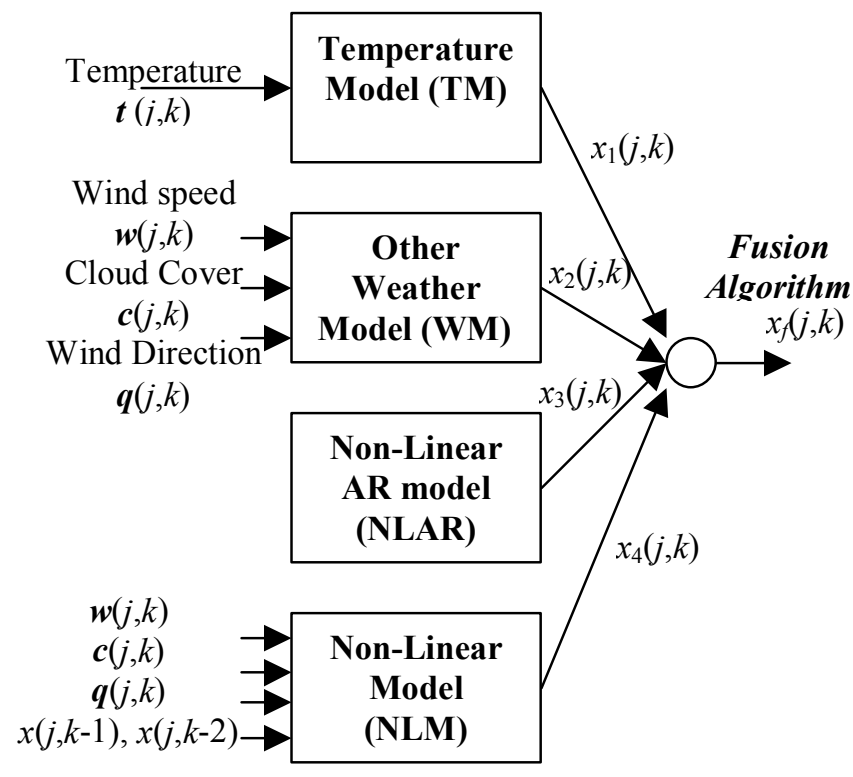

Figure 4. Data fusion model overview

The sub-models all use feed forward neural networks. The network structure determination and input selection procedures are similar to those explained in detail in [6].
The Temperature Model (TM) input, $\boldsymbol{t}(j, k)$, is a vector of the previous 72 hours of temperature from hour $j$ on day $k$. Similarly the other Weather Model (WM) uses vectors of wind speed, $\boldsymbol{w}(j, k)$, cloud cover, $\boldsymbol{c}(j, k)$, and wind direction, $\boldsymbol{q}(j, k)$ for the previous 72 hours of weather. The Non-Linear Auto-Regressive model (NLAR) uses the previous 2 days of residual, $x(j, k-1)$ and $x(j, k-2)$. The Non-Linear Model (NLM) uses all the available inputs.

\subsection{Fusion algorithm}

The data fusion algorithm described in [10] seeks to minimize the variance of the fused forecast based on the covariance matrix of the sub-model forecasts. The cross-covariance of the forecasts is considered and the distribution of the forecast error noise is not restricted to Gaussian but merely required to be unbiased. A combined forecast, $x_{f}(j, k)$, of the load is created using a weighted average of the individual forecasts $x_{1}(j, k), \ldots, x_{4}(j, k)[10]$ :

$$
x_{f}(j, k)=\sum_{i=1}^{4} A_{i}(j) x_{i}(j, k)
$$

where $A_{i}(j)$ is the weight applied to the forecast from submodel $i$ for hour $j$, and is derived from the error covariance matrices of $x_{1}(j, k), \ldots, x_{4}(j, k)$ as:

$$
\left[A_{1}(j) A_{2}(j) A_{3}(j)\right]=\left[P_{4,1}^{\prime}(j) P_{4,2}^{\prime}(j) P_{4,3}^{\prime}(j)\right] \boldsymbol{P}^{-1}
$$

where $P_{4,1}^{\prime}(j), P_{4,2}^{\prime}(j), P_{4,3}^{\prime}(j)$ and $\boldsymbol{P}$ are auxiliary variables derived from the error covariance of $x_{1}(j, k), \ldots, x_{4}(j, k)$ :

$$
P_{i, n}(j)=\frac{1}{M} \sum_{k=1}^{M}\left(x(j, k)-x_{i}(j, k)\right)\left(x(j, k)-x_{n}(j, k)\right)
$$

where $P_{i, n}(j)$ is the error covariance of sub-model $i$ with submodel $n$ for hour $j$. The auxiliary variables are then defined as:

$$
P_{4, i}^{\prime}(j)=P_{4,4}(j)-P_{4, i}(j) \quad i \neq 4
$$

and

$$
\boldsymbol{P}=\left[\begin{array}{ccc}
P_{1,1}^{\prime}(j) & P_{1,2}^{\prime}(j) & P_{1,3}^{\prime}(j) \\
P_{2,1}^{\prime}(j) & P_{2,2}^{\prime}(j) & P_{2,3}^{\prime}(j) \\
P_{3,1}^{\prime}(j) & P_{3,2}^{\prime}(j) & P_{3,3}^{\prime}(j)
\end{array}\right]
$$

where

$$
P_{i, n}^{\prime}(j)=P_{i, n}(j)-P_{4, n}(j)-P_{i, 4}(j)+P_{4,4}(j) \quad i \neq 4, n \neq 4
$$

The final weight $A_{4}$ is determined using the constraint that $x_{f}(j)$ is unbiased:

$$
A_{4}(j)=1-\sum_{i=1}^{3} A_{i}(j)
$$

Finally the fused load $y_{f}(i, j)$ is estimated by reintroducing the trend: 


$$
y_{f}(j, k)=d(j, k)+x_{f}(j, k)
$$

\section{Results}

Figure 5 below shows the Mean Absolute Percentage Error $(\mathrm{MAPE})^{*}$ for the sub-models and the fusion model using actual weather inputs in the novelty set. The fusion weights are calculated using the error covariance matrices of the submodels over the training set with actual weather inputs. As can be seen the fusion model performs best for each hour of the day.

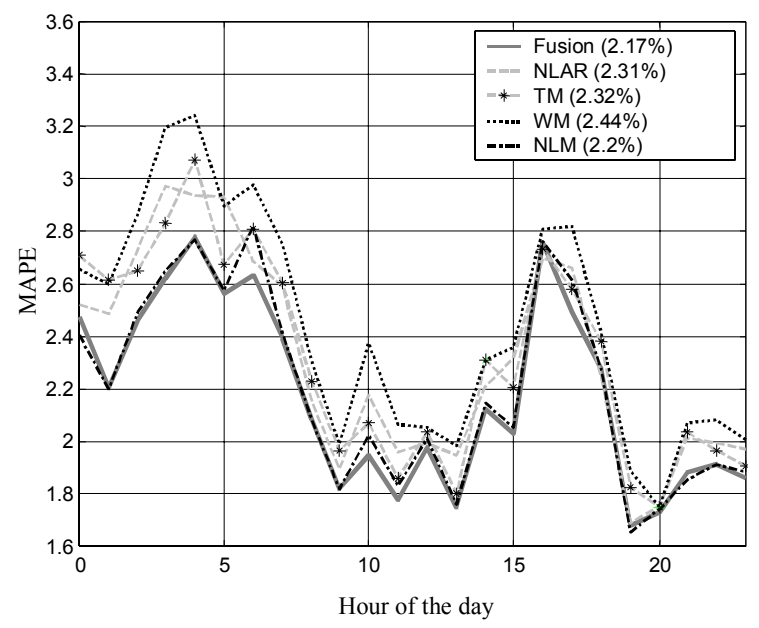

Figure 5. MAPE as a function of hour of the day for fusion and sub-models (notes: novelty set, actual weather used)

Table 4 below, summarise's the results in the training, validation and novelty data sets.

\begin{tabular}{|c|l|l|l|}
\hline Model & $\begin{array}{c}\text { Training } \\
\text { Set (\%) }\end{array}$ & $\begin{array}{c}\text { Validation } \\
\text { Set (\%) }\end{array}$ & $\begin{array}{c}\text { Novelty } \\
\text { Set (\%) }\end{array}$ \\
\hline NLAR & 2.30 & 2.17 & 2.31 \\
\hline TM & 2.31 & 2.15 & 2.32 \\
\hline WM & 2.39 & 2.22 & 2.44 \\
\hline NLM & 2.21 & 2.08 & 2.20 \\
\hline Fusion & $\mathbf{2 . 1 9}$ & $\mathbf{2 . 0 7}$ & $\mathbf{2 . 1 7}$ \\
\hline
\end{tabular}

Table 4. The MAPE's of the models using actual weather inputs.

Figure 6 below shows the Mean Absolute Percentage Error (MAPE) for the sub-models and the fusion model using pseudo-forecast weather inputs in the novelty set. The effect of weather forecast errors are now accounted for by calculating the error covariance matrices of the sub-models over the training set with pseudo-weather forecast inputs. As can be seen the fusion model again performs best for each hour of the day.

\footnotetext{
* The MAPE is the standard error measure in the field of STLF as it allows comparison between systems.
}

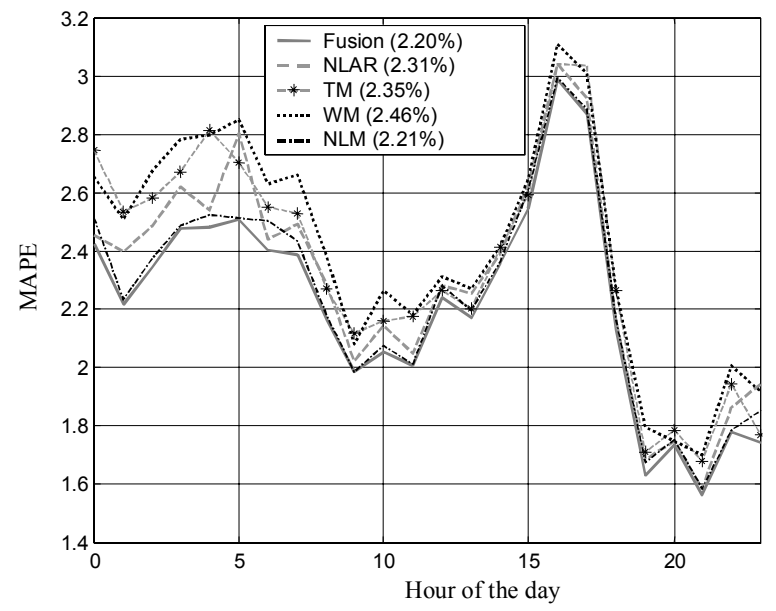

Figure 6. MAPE as a function of hour of the day for fusion and sub-models (notes: novelty set, pseudo-weather forecasts used)

\begin{tabular}{|c|l|l|l|}
\hline Model & $\begin{array}{c}\text { Training } \\
\text { Set (\%) }\end{array}$ & $\begin{array}{c}\text { Validation } \\
\text { Set (\%) }\end{array}$ & $\begin{array}{c}\text { Novelty } \\
\text { Set (\%) }\end{array}$ \\
\hline NLAR & 2.30 & 2.17 & 2.31 \\
\hline TM & 2.36 & 2.20 & 2.35 \\
\hline WM & 2.40 & 2.24 & 2.46 \\
\hline NLM & 2.25 & 2.12 & 2.21 \\
\hline Fusion & $\mathbf{2 . 2 2}$ & $\mathbf{2 . 1 0}$ & $\mathbf{2 . 2 0}$ \\
\hline
\end{tabular}

Table 5. The MAPE's of the models using pseudo weather forecast inputs.

Comparing Tables 4 and 5, it can be seen that the NLAR models are unaffected by weather forecast errors as they have no weather inputs. The other sub-models deteriorate with the inclusion of pseudo-weather forecast errors. The fusion model deteriorates with the inclusion of pseudo-weather forecast error but maintains it's position as the best model.

\section{Conclusion}

This paper examined the effect of weather forecast errors in load forecasting models. In Section 3, the distribution of the weather forecast errors was examined and it was found that a Gaussian distribution was not appropriate in this case. Rather, a structure exists which means that the weather forecast error will have a large effect on any aggregate weather variables.

The structure of the weather forecast errors was then used to produce pseudo-weather forecast errors from 1986 to 2000 which have the accuracy of current weather forecasts. This is important as, for example, weather forecasts from 1986 are less accurate than current weather forecasts and thus of no relevance in predicting future loads.

A model fusion technique was then proposed for minimising the effect of weather forecast errors. In general weather forecast error causes approximately $1 \%$ deterioration in load forecasts of all models used here. This figure, though 
important, is not as high as suggested by [5] and [8], for their systems. However, the fusion model was capable of adjusting the weighting of the sub-models to reflect that the weather based sub-models deteriorated relative to the AR model. Finally, the fusion model was shown to successfully separate the tasks of model training and rejecting weather forecast errors.

\section{Acknowledgements}

The authors would like to express their thanks to Eirgrid for the availability of data and their continued support and advice.

\section{References}

[1] J.M. Bates, C.W.J. Granger, "The combination of forecasts", Operational Research Quarterly, 20, pp. 451-468, (1969).

[2] P.J. Brockwell, R.A. Davis, Time Series: Theory and Methods, New York USA: Springer Verlag Inc, (1987).

[3] S.T. Chen, D.C. Yu, A.R. Moghaddamjo, "Weather sensitive short-term load forecasting using non-fully connected artificial neural network", IEEE Transactions on Power Systems, 7 (3), pp. 1098-1104, (1992).

[4] P.K. Dash, H.P. Satpathy, A.C. Liew, S. Rahman, "A realtime short-term load forecasting system using functional link network", IEEE Transactions on Power Systems, 12 (2), pp. 675-681, (1997).

[5] A.P. Douglas, A.M. Breipohl, F.N. Lee, R. Adapa, "The impacts of temperature forecast uncertainty on Bayesian load forecasting", IEEE Transactions on Power Systems, 13 (4), pp. 1507-1513, (1998).

[6] D. Fay, J.V. Ringwood, M. Condon, M. Kelly, "24-hour electrical load data - a sequential or partitioned time series?", Neurocomputing, 55 (3-4), pp. 469-498, (2003).

[7] S.H. Hippert, C.E. Pedriera, R.C. Souza, "Neural networks for short-term load forecasting: a review and evaluation", IEEE Transactions on Power Systems, 16 (1), pp. 44-55 (2001).

[8] IEEE committee report, "Problems associated with unit commitment in uncertainty", IEEE Transactions on Power Apparatus and Systems, 104 (8), pp. 2072-2078. (1985).

[9] P.A. Mastorocostas, J.B. Theocharis, S.J. Kiartzis, A.G. Bakisrtzis, "A hybrid fuzzy modeling method for short-term load forecasting", Mathematics and Computers in Simulation, 51, pp. 221-232, (2000).

[10] H. McCabe, "Minimum trace fusion of $N$ sensors with arbitrary correlated sensor to sensor errors", in: Proceedings, IFAC Conference on Distributed Intelligent Systems, Virginia, USA, August 1991, pp. 229-234. (1991).
[11] T. Miyake, J. Murata, K. Hirasawa, "One-day through seven-day-ahead electrical load forecasting in consideration of uncertainties of weather information", Electrical Engineering in Japan, 115 (8), pp. 135-142. (1995).

[12] I. Moghram, S. Rahman, "Analysis and evaluation of five short-term load forecasting techniques", IEEE Transactions on Power Systems, 4 (4), pp. 1484-1491, (1989).

[13] A.K. Palit, D. Popovic, "Nonlinear combination of forecasts using artificial neural network, fuzzy logic and neuro-fuzzy approaches", in: Proceedings, IEEE international conference on fuzzy systems, 2, pp. 566-571, (2000).

[14] A.D. Papalexopoulos, C.T. Hesterburg, "A regression based approach to short term system load forecasting", IEEE Transactions on Power Systems, 5 (4), pp. 1535-1547, (1990).

[15] D.C. Park, M. El-Sharkawi, R. Marks, "Electric load forecasting using an artificial neural network", IEEE Transactions on Power Systems, 6 (2), pp. 442-449, (1991).

[16] D. Park, O. Mohammed, A. Azeem, R. Merchant, T. Dinh, "Load curve shaping using neural networks", in: Proceedings, Second International Forum on Applications of Neural Networks to Power Systems, Yokohama, Japan, April 1993, IEEE, pp. 290-295, (1993).

[17] D.J. Reid, "Combining three estimates of gross domestic products", Economica, 35, pp. 431-444, (1968).

[18] L. Rong, W. Ping, H. Wenlei, , "A novel method for wine analysis based on sensor fusion technique", Sensors and Actuators, B 66, pp. 246-250, (2000).

[19] M. Tamimi, R. Egbert, "Short term electric load forecasting via fuzzy neural collaboration", Electric Power Systems Research, 56, pp. 243-248, (2000).

[20] L. Xiong, A.Y. Shamseldin, K.M. O'Connor, "A nonlinear combination of the forecasts of rainfall-runoff models by the first order Takagi-Sugeno fuzzy system", Journal of Hydrology, 245, pp. 196-217, (2001).

[21] H. Yoo, R.L. Pimmel, "Short-term load forecasting using a self-supervised adaptive neural network", IEEE Transactions on Power Systems, 14 (2), pp. 779-784, (1999). 